\title{
RIESGOS ERGONÓMICOS DE LOS PROFESIONALES DE ENFERMERIA QUE LABORAN EN LAS AREAS CRITICAS DE LOS HOSPITALES DEL MINSA Y ES-SALUD DEL DEPARTAMENTO DE ICA -2009
}

\section{ERGONOMIC RISKS OF NURSING PROFESSIONALS WORKING IN CRITICAL AREAS IN THE MINSA AND ESSALUD HOSPITALS IN THE DEPARTMENT OF ICA - 2009}

\author{
Olga M. Curro Urbano ${ }^{1, a, c}$. Marianela Pecho Tataje ${ }^{2, a, b}$ Viviana Loza Félix $^{3, a}$ \\ Teresa Carbonel Ramírez ${ }^{4, a}$ Diana Calle Jacinto ${ }^{5, a}$
}

1.- Facultad de Enfermería, Universidad Nacional San Luis Gonzaga. Ica, Perú. 2.- Hospital Félix Torrealva Gutiérrez, EsSalud. Ica, Perú. 3.- Hospital Regional MINSA. Ica, Perú. 4.- Hospital Arzobispo Loayza MINSA. Lima Perú. 5.- Clínica Internacional. Lima, Perú

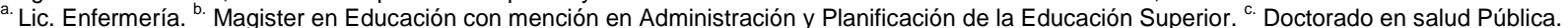

\section{RESUMEN}

Objetivo: Identificar los riesgos ergonbómicos a los que los profesionales de enfermería, que trabajan en áreas críticas de hospitales del MINSA y ESSALUD-, están expuestos. Se incluyeron enfermeras que trabajan en los servicios críticos (salas de emergencia y de operaciones, unidades de cuidados intensivos e intermedios y las unidades de hemodiálisis) que realizan atención directa al paciente adulto hospitalizado. Material y Métodos: Se utilizó el método descriptivo de corte transversal. La población estuvo conformada por las enfermeras de los hospitales de Ica. Se utilizó un cuestionario para evaluar los riesgos ergonómicos a los que fueron expuestas. La muestra consistió en 59 (40,7\%) profesionales de enfermería de los hospitales del MINSA y 86 (59,3\%) enfermeras de los hospitales de ESSALUD. Resultados: El análisis sociodemográfico mostró un predominio del sexo femenino en los hospitales del MINSA (50; 34,5\%) y ESSALUD (84; 57,9\%), predominaron las edades 46 a 50 años en las enfermeras del MINSA (18; 12,4\%) y 51-55 años en las enfermeras de ESSALUD (22; 15,2\%). El tiempo de servicio en la institución es de 11-15 años de enfermeras de hospitales del MINSA $(17 ; 11,7 \%)$ y de 6 a 10 años en los hospitales de ESSALUD (28; 19,3\%). Los resultados muestran que las enfermeras (MINSA: 38; 26,2\% ESSALUD: 57 ; 39,3\%), tienen dolor leve en la espalda por estar de pie durante más de 06 horas (valor de Pearson Chi cuadrado $=7,793$ y el valor $p=0,05 S$ ); también (MINSA: 30; 20,7\% ESSALUD: $52 ; 35,9 \%$ ) tienen dolor leve en la espalda al levantar cargas superiores a $15 \mathrm{~kg}$ (valor de Pearson Chi cuadrado $=11,629$ y el valor de $p<0,05 \mathrm{~S}$ ). Por otra parte, las enfermeras (MINSA: 44; 30,3\% - ESSALUD: 47; 32,4\%) presentan dolor cervical leve cuando se movilizan los pacientes (valor de Pearson Chi cuadrado $=0,603$ y el valor de $p<0,05 \mathrm{~S}$ ). Conclusiones: La mejora de las condiciones de trabajo mediante la incorporación de procedimientos de control de prevención es una necesidad.

Palabras clave: Ergonomía y enfermera.

\section{SUMMARY}

Objective: Identify ergonomic risks to which the professional nurses, working in critical areas of MINSA-and-ESSALUD hospital's, are exposed. Nurses working in critical services (emergency and operating rooms, intensive and intermediate care units, and hemodialysis units) performing direct care to hospitalized adult patient were included. The descriptive cross-sectional method was used. The population consisted of nurses from hospitals in Ica. Material and Methods: A questionnaire was used to assess ergonomic risks to which they were exposed. The sample consisted of 59 (40.7\%) nurses from MINSA hospitals and 86 (59,3\%) nurses from ESSALUD HOSPITALS. Results: The socio-demographic analysis showed a female predominance in hospitals from MINSA (50; 34,5\%) and ESSALUD (84; 57,9\%), predominance of ages $46-50$ years in MINSA's nurses 
(18; 12,4\%) and 51-55 years in ESSALUD's nurses (22; 15,2\%). The length of service in the institution is $11-15$ years in nurses from the MINSA hospital's $(17 ; 11,7 \%)$ and $6-10$ years in ESSALUD hospital's ones (28; 19,3\%). The results shows that nurses (MINSA: 38; $26,2 \%$ ESSALUD: $57 ; 39,3 \%$, have mild back pain because of being stand up for more than 06 hours (Pearson's Chi Square value $=7,793$ and $p$ value $=0,05 \mathrm{~S}$ ); also, they (MINSA: 30; 20,7\% ESSALUD: 52; 35,9\%) have mild back pain when lifting loads greater than 15kg (Pearson's Chi Square value $=11,629$ and $p$ value $<0,05 \mathrm{~S}$ ). Moreover, nurses (MINSA: 44; 30,3\% - ESSALUD: 47; $32.4 \%$ ) have mild cervical pain when they are mobilizing patients (Pearson's Chi Square value $=$ 0,603 and $p$ value $<0,05 \mathrm{~S}$ ). Conclusions: Improving the working conditions by incorporation of preventive control procedures is a needing.

Keywords: Ergonomics and nurse.

\section{INTRODUCCION}

Riesgos ergonómicos son aquellos directamente ligados al diseño de equipos, al estrés, cargas de trabajo, fatiga y trabajo repetitivo. Los factores de riesgo ergonómico, se refieren a las características del ambiente de trabajo que causa un desequilibrio entre los requerimientos del desempeño y la capacidad de los trabajadores en la realización de las tareas. Estos factores de riesgo están asociados con la postura, la fuerza, el movimiento, las herramientas, los medios de trabajo y el entorno laboral. Los problemas ergonómicos se manifiestan en desgastes y daños debidos a sobrecargas o posturas incorrectas de trabajo. Los trastornos músculoesqueléticos constituyen un importante problema para el personal de enfermería, quienes presentan una tasa relativamente alta de síndrome doloroso lumbar, síntomas neurológicos y algias de esfuerzo.

La OMS (2003) considera que en América Latina y el Caribe sólo se notifican entre el $1 \%$ y el $5 \%$ de enfermedades ocupacionales, y que los trabajadores de salud están expuestos a una considerable variedad de riesgos (OPS 2003) (1). Los Centros asistenciales generalmente tienen por finalidad proteger a los usuarios o clientes, y no se considera al personal que labora. En el Convenio No149 de la Organización Internacional de Trabajo (OIT) relacionado al "Empleo y las condiciones de trabajo y de Vida del personal de enfermería", (1997) solicita a los estados integrantes que mejoren las leyes y reglamentos, sobre seguridad e higiene en el trabajo, con carácter especial del trabajo de enfermería y del medio en que ejerce (2).

El esfuerzo físico es parte esencial de algunas actividades laborales, no sólo es un componente de los trabajos «pesados» (minería, construcción,), sino que es un elemento de fatiga importante, aunque menos evidente, que en otros trabajos como mecanografía, enfermería, montaje de pequeñas piezas, confección. A veces el mantenimiento de una misma postura (de pie o sentado) durante ocho horas puede ser causa de lesiones corporales. En España, los accidentes de trabajo por sobreesfuerzos constituyen el $25 \%$ del total de notificados. En 1998 , el $74,3 \%$ de las enfermedades profesionales notificadas fueron debidas a procesos músculo-esqueléticos y en la Encuesta Nacional de Condiciones de Trabajo realizada en 1999 , un $76,8 \%$ de las personas entrevistadas manifestaron sentir alguna molestia músculoesquelética debido a posturas y esfuerzos derivados del trabajo (3). La realización de movimientos rápidos de forma repetida, y que no representen un gran esfuerzo físico (empaquetado, mecanografía), el mantenimiento de una postura que suponga una contracción muscular continua de una parte del cuerpo (mobiliario o herramientas inadecuadas), o la realización de esfuerzos más o menos bruscos con un determinado grupo muscular (amasar) y la manipulación manual de cargas, pueden 
generar alteraciones por sobrecarga en las distintas estructuras del sistema osteomuscular: al nivel de hombros, el cuello y los miembros superiores (3).

Según Rescalvo (2004) existen tres tipos de técnicas de prevención, la técnica de prevención primaria: (..) suprimen los factores desfavorables antes que generen la enfermedad 0 accidente (...) eliminan los factores ambientales de riesgo (...) educa en la salud y crea hábitos saludables. La técnica de prevención secundaria es cuando aborda la enfermedad en fase incipiente, aún en fase reversible, por medio del diagnóstico precoz y la técnica de prevención terciaria (...) elimina los efectos de las secuelas de la enfermedad cuando esta ya se ha desarrollado (4).

En el presente trabajo se planteó como objetivo: identificar los riesgos ergonómicos a los que se expone el personal profesional de enfermería, que laboran en las áreas críticas de los hospitales del Minsa y Esalud del departamento de Ica.

\section{MATERIAL Y METODO}

Para valorar los niveles de dolor se empleó la escala categórica de Arecon A, el al (2004). Según el objetivo planteado y el alcance de los resultados, el tipo de estudio más apropiado para abordar la problemática fue el descriptivo y de corte transversal.

\section{Población y Muestra}

La población motivo de estudio fueron las enfermeras y los enfermeros que desempeñan su labor en los Hospitales del MINSA: Hospital Regional, Hospital Santa María del Socorro y las de Essalud: Hospital Félix Torrealva Gutiérrez N III del departamento de Ica en los servicios de: Emergencia, Unidad de Cuidados Intensivos., Unidad de Cuidados Intermedios, Unidad de Vigilancia Intensiva, Centro quirúrgico y Hemodiálisis. El número total de encuestados para el presente estudio fueron 145. Dada la disponibilidad de recursos para el manejo de datos, no se trabajó con muestra.

\section{RESULTADOS}

Los resultados obtenidos nos demuestran que las enfermeras del MINSA presentan dolor leve de espalda al permanecer de pie por más de 06 horas, según respondieron 38 $(26,2 \%)$ y las de Es-Salud en número de 57 $(39,3 \%)$ (Tabla 1). En los Hospitales del MINSA manifestaron dolor dorsal leve al manipular cargas superiores a $15 \mathrm{Kg}$ en 30 encuestadas que hacen el $(20,7 \%)$ y en EsSalud 52 enfermeras respondieron, alcanzando $\quad(35,9 \%) \quad($ Tabla 2). Las enfermeras encuestadas del MINSA y EsSALUD, presentaron dolor cervical leve al movilizar pacientes, siendo los resultados a nivel del MINSA un porcentaje de $30,3 \%$ de 44 y en Es-SALUD 32,4\% de 91 enfermeras (Tabla 3).

Tabla 1. Riesgos Ergonómicos de los Profesionales de Enfermería por presentar dolor dorsal cuando permanece de pie mayor a 06 horas, según entidad donde laboran.

\begin{tabular}{|c|c|c|c|c|c|c|}
\hline \multirow[t]{3}{*}{ DOLOR DORSAL } & \multicolumn{4}{|c|}{ ENTIDAD } & \multicolumn{2}{|c|}{ TOTAL } \\
\hline & \multicolumn{2}{|c|}{ MINSA } & \multicolumn{2}{|c|}{ ESSALUD } & & \\
\hline & $\mathrm{N}^{\mathrm{a}}$ & $\%$ & $\mathrm{~N}^{\mathrm{a}}$ & $\%$ & $\mathrm{~N}^{\mathrm{a}}$ & $\%$ \\
\hline Leve & 38 & 26.2 & 57 & 39,3 & 95 & 65,5 \\
\hline Moderado & 18 & 12,4 & 14 & 9,7 & 32 & 22,1 \\
\hline Intenso & 0 & 0,0 & 2 & 1,4 & 2 & 1,4 \\
\hline No presenta dolor & 3 & 2,1 & 13 & 9,0 & 16 & 11,0 \\
\hline TOTAL & 59 & 40.7 & 86 & 59.3 & 145 & 100.0 \\
\hline
\end{tabular}


Tabla 2. Riesgos ergonómicos de los profesionales de enfermería por presentar dolor dorsal cuando levanta cargas de $15 \mathrm{Kg}$, según entidad donde laboran

\begin{tabular}{lrrrrrr} 
& \multicolumn{4}{c}{ EOLOR DORSAL } & \multicolumn{3}{c}{ ENTIDAD } & \multicolumn{2}{c}{ TOTAL } \\
\hline & \multicolumn{1}{c}{ MINSA } & ESSALUD & & \\
& № & $\%$ & \multicolumn{1}{c}{$\mathrm{N}^{\mathbf{a}}$} & $\%$ & \multicolumn{1}{c}{ № } & \multicolumn{1}{c}{$\%$} \\
Leve & $\mathbf{3 0}$ & $\mathbf{2 0 , 7}$ & $\mathbf{5 2}$ & $\mathbf{3 5 , 9}$ & $\mathbf{8 2}$ & $\mathbf{5 6 , 6}$ \\
Moderado & 23 & 15,9 & 16 & 11,0 & 39 & 26,9 \\
Intenso & 3 & 2,1 & 2 & 1,4 & 5 & 3,4 \\
No presenta dolor & 3 & 2,1 & 16 & 11,0 & 19 & 13,1 \\
\hline TOTAL & 59 & 40,7 & 86 & 59,3 & 145 & 100,0 \\
\hline
\end{tabular}

Tabla 3. Riesgos Ergonómicos de los profesionales de Enfermería por presentar dolor cervical cuando moviliza pacientes según entidad donde laboran

\begin{tabular}{|c|c|c|c|c|c|c|}
\hline \multirow[t]{2}{*}{ DOLOR CERVICAL } & \multicolumn{4}{|c|}{ ENTIDAD } & \multicolumn{2}{|c|}{ TOTAL } \\
\hline & & NSA & & ;ALUD & & \\
\hline & $\mathrm{N}^{\mathrm{a}}$ & $\%$ & $\mathrm{~N}^{\mathrm{a}}$ & $\%$ & $\mathrm{~N}^{\mathrm{a}}$ & $\%$ \\
\hline Leve & 44 & 30,3 & 47 & 32.4 & 91 & 62,8 \\
\hline Moderado & 8 & 5,5 & 12 & 8,3 & 20 & 13,8 \\
\hline Intenso & 1 & 0,7 & 0 & 0,0 & 1 & 0.7 \\
\hline No presenta dolor & 6 & 4,1 & 27 & 18,6 & 33 & 22,8 \\
\hline TOTAL & 59 & 40,7 & 86 & 59,3 & 145 & 100,0 \\
\hline
\end{tabular}

\section{DISCUSION}

Se encuentra también coincidencia con el estudio realizado por Marras (1999)citado en Briseño, donde el $64 \%$ del personal de enfermería sufre dolores de espalda (5). Coincide igualmente en el estudio realizado por Castillo F. en 1977(citado por Briseño), donde se detectaron dolencias en la columna vertebral en las enfermeras, atribuyéndoles a los ascensos y descensos de escaleras y las largas horas de pie (5). Delhin et al.(1976) citado en Briseño; refiere que el $46,8 \%$ de 267 enfermeras de un hospital geriátrico de Suecia, tienen dolor de espalda (6). Stubbs, en (1983) citado por Briseño; realizó una investigación en 3.912 enfermeras del Servicio Nacional de Salud de Gran Bretaña, y encontró que un $43.1 \%$ ha sufrido dolores en la espalda, llegó también a la conclusión que el personal de enfermería padece de dorsalgias, algias de esfuerzo y síntomas neurológicos (6). Armengol et al. citado en
Briseño; en su trabajo de investigación sobre prevención de las lesiones de espalda en el trabajo hospitalario, refieren que el personal sanitario es uno de los grupos de alto riesgo de lesiones de espalda; en el estudio que se realizó en el Hospital de Mutua de Terrassa en 181 profesionales de enfermería, el 64\% (115 enfermeras) refiere que alguna vez sufrió de dolor de espalda, y aquellas que sus edades estaban comprendidas entre 20 a 30 años de edad, habían sufrido de dolor de espalda en un $67 \%$ (5). Según Gore Donald (2001) citado por Briseño en el trabajo de investigación de Valoración de los pacientes con dolor cervical, refiere que la prevalencia del dolor cervical de origen no traumático en los EE.UU es del 10\% (5). 


\section{CONCLUSIONES}

1.- Los mayores riesgos que presentan los profesionales de enfermería que trabajan en los Hospitales del MINSA Y ES-SALUD es dolor leve de espalda al permanecer por más de 06 horas de pie, también presenta dolor leve dorsal al levantar cargas mayores de $15 \mathrm{Kg}$ y finalmente presentan dolor cervical leve al movilizar pacientes.

2.- Consideramos que las autoridades de las instituciones de salud de la región, deben valorar los riesgos ergonómicos del personal de salud.

3.- Llegamos a la conclusión de que la mejora de las condiciones de trabajo mediante la incorporación de procedimientos de control de prevención es una necesidad.

\section{Correspondencia}

Olga Curro-Urbano

Correo electrónico: olgamcu_inv@yahoo.es

\section{REFERENCIAS BIBLIOGRAFICAS:}

1. Organización Panamericana de la Salud (OPS). Washington .D.C 25 de abril del 2003. Disponible en: http://www.estruc plan. com.ar/rss.asp OIT.

2. Organización Internacional del Trabajo (OIT). Convenio 149 sobre el Personal de Enfermería, empleo y condiciones de trabajo y de vida. Sesión de la
Conferencia :63.Ginebra, Suiza. 11:07 1979

3. Almodovar A. III Encuesta Nacional de Condiciones de trabajo. Código ET 082. Madrid, España 1999.

4. Rescalvo S. Ergonomía y salud. Consejería de economía y empleo. Dirección de trabajo y prevención de riesgos laborales. Madrid, España. p.32.2004

5. Briseño C. Estudio de riesgos ergonómicos y satisfacción laboral. Revista de salud Pública Rv. Vol IX Número 1, 2005. Mar de Plata. Argentina. Disponible en: http://www.laenfermerahoy.com.ar/articulo .php?id=25

6. Briseño C. Riesgos ergonómicos en el personal de enfermería. Hospital Ángel C. Padilla - Tucumán Argentina. Disponible en:

http://www.portalesmedicos.com/publicaci ones/articles/19/1/Riesgos-ergonomicosen-el-personal-deenfermeria.html

Recibido: 23/02/14

Aprobado para Publicación: 29/05/14 